\title{
On the Concept of Flexibility: A Dual Control Perspective
}

\author{
ACJ DE LEEUW \\ University of Groningen, Groningen, The Netherlands \\ HW VOLBERDA \\ Erasmus University, Rotterdam, The Netherlands \\ (Received July 1995; accepted after revision October 1995)

\begin{abstract}
Organizational flexibility has recently received much attention from researchers, management consultants and practitioners. In general, the term 'flexibility' has a positive connotation: flexible organizations are the better ones. However, the meaning of flexibility as well as its relation to the functioning of an organization is still ambiguous. This article develops a systematic and multidimensional picture of flexibility on the basis of views taken from the systems theory of control. The general idea is that flexibility can be analysed as a characteristic of some dual and relative control relation between the organization and its environment, which fosters organizational independence. It is argued that flexibility is a function of the control capability of the management as well as the controllability of the organization. Flexibility is thus concerned with the promotion of the management's control capability or competence. The management's ability to allocate and use this capacity with success, however, is dependent upon organizational conditions that determine the controllability. The organization design task, therefore, involves the creation of appropriate organizational conditions to foster flexibility. This article clarifies the paradoxical nature of flexibility and the existence of different types of flexibility. Copyright $(\mathbb{C}) 1996$ Elsevier Science Ltd
\end{abstract}

Key words-flexibility, control, design, cybernetics, systems, strategy

\section{INTRODUCTION}

ORGANIZATIONAL FLEXIBILITY has recently once more recieved much attention from researchers, management consultants and practitioners. Intuition would suggest that organizational flexibility, which roughly means mobility, responsiveness, agility, suppleness or litheness, is important and desirable. Yet intuitive understanding alone is not a solid enough basis for analysis and design. Nearly 20 years ago, Steers [48] demonstrated, on the basis of seventeen organizational effectiveness studies, that flexibility was the evaluation criterion mentioned most frequently. However, the added value of the construct to the theory and practice of management is in many cases very restricted $[52,53]$. We may rightfully ask ourselves if flexibility is used as a magic word or belongs to a new business fad [26]. What does flexibility really mean? Is flexibility required by every organization as some new 'one best way'? What makes organizations flexible? In contrast with the importance of such questions, the meaning of flexibility and its relation to the functioning of an organization is still ambiguous. A critical and unbiased approach is lacking. In this article, we will contribute to such an approach by developing a clearer and more encompassing concept of flexibility.

We wish to make clear that our approach takes a specific functionalistic and systems 
theoretical stand; we aim to contribute to methods of analysis and the improvement of organizational functioning. In short, we will look at organizations from a functionalistic perspective [10].

Our analysis started with the intuitive idea of flexibility as an important aspect of a dual and relative control relation between the organization and its environment: flexible organizations are open systems but they are not puppets in the hands of their environments. They are able to free themselves, to some extent, from environmental control. This intuitive idea led quite naturally to the choice of the systems theory of control as one of the conceptual theories for analysing flexibility. Among other things, this theory enables us to analyse to what extent control is possible and which factors determine or impede its success. We will show that, from this perspective, two questions have to be answered: "What makes an organization uncontrollable from the environment?" and "What makes an organization powerful in controlling its environment?"

In the next section we shall start with the clarification of some main ideas from this systems theory of control. We will then gradually develop our concept of flexibility and will finally show that flexibility is a multidimensional concept of a paradoxical nature related to stability. Flexibility is not only a management task but is also a task of organization design. Among other things, it will be argued that different sets of specific circumstances ask for different types of flexibility.

\section{SYSTEMS THEORY OF CONTROL: ITS FUNDAMENTAL PRINCIPLES AND UNDERLYING PARADIGM}

The use of systems theory in order to define the concept of flexibility may lead to misunderstanding, because there are many different

'The philosophical systems movement presupposes that organizations are systemic in nature, while the organic systems movement assumes that organizations are living organisms which strive for survival, growth, and exchange of material with the surrounding environment. For a more elaborate discussion, see De Leeuw [15, pp. 63-65].

${ }^{2}$ For a more elaborate discussion of the systems theory of control and some of its central concepts we refer to, for instance, several of the works of De Leeuw [14-17], Kickert and Van Gigh [27] and Beer [9]. systems approaches [23]. The systems theory of control is part of the conceptual systems approach [15, pp. 63-68], which may be seen in the tradition of the conceptual and methodological views of Ackoff [3,4], Ashby's foundations of cybernetics [8], and the ideas of self-organization and 'autopoiesis' of, for example, Varela [22, 32]. In contrast with philosophical and organic systems approaches, ${ }^{\prime}$ the conceptual systems approach is not an empirical theory or model but rather a methodology [23]. Snow [47], working along the lines of Ackoff, argues that system theory may even bridge the gap between the functionalistic and interpretative approaches in organization theory.

According to De Leeuw $[15$, p. 66], the conceptual systems approach offers various systems concepts and modes of thought in terms of instruments and tools for application in real-life situations. It is, so to speak, a modelling tool-box that we will use for modelling organizational flexibility. The most fundamental decision when applying systems theory then is the making of distinctions, the very act of defining the system concerned, of distinguishing it from its environment. Kickert [26, p. 485] concludes that the advantage of this conceptual approach to organizational flexibility is that concepts are abstract and empirically vacuous, and accordingly less limited to their area of application, while in addition they are not vague but unequivocal.

On the other hand, De Leeuw [15, p. 64] rightly notices that this abstract nature also brings with it some major problems. The conceptual systems approach demands much practice and experience in modelling systems.

In this article, the concepts of the systems theory of control are only defined roughly as a reminder, for they are defined thoroughly elsewhere. ${ }^{2}$ Although it is possible to state very precise mathematical definitions in connection with the systems theory of control, we will only use relatively simple concepts and omit any unnecessarily complicated mathematics.

\section{The paradigm of control}

At the heart of the systems theory of control there is the paradigm of control: a set of concepts for looking at and analysing parts of reality. Of course, a central concept is 'control'. The problem with the word control is that it can 
easily be misunderstood as tied to the classical hierarchical mode of management, which is not implied by the specific meaning of the word control in this theory. In the same way, our concept of control does not have the meaning of close monitoring and regular checks that it has in the field of accounting. Comparable risks of misunderstanding arise when using other verbs such as steering and regulating.

The systems theory of control originates from a general definition of control: any manner of directed influence ${ }^{3}[14,16]$. We may recall that originators of cybernetics like Wiener and Ashby never thought of control in the sense of a strict determination of processes. The label 'control' and its use to describe aspects of an organization depends on the viewpoint of the observer explaining phenomena as such. There is not a definitional need for the success of this directed influence. As we all know, much control is not successful, and can even be counterproductive. The idea of control is thus not defined by its success but by the ascribed intention.

This paradigm clearly enables us to recognize that the function of management (as a process) is to control in terms of producing directed influence. Nonetheless, it also allows for the analysis of reasons for not being successful, as a result of, for example, the limited predictability, the active and reactive nature of organizational reality and the non-linearity of circles of influence. The theory enables us to analyse situations involving the so-called illusion of control: even control that is not successful at all is nevertheless called control. As has been said before, it must not be confused with an absolute concept of control that leaves no room for the controlled system. The relative concept of control facilitates its application for the analysis of a huge variety of forms of directed influence, such as power

\footnotetext{
${ }^{3}$ We seriously thought about introducing a new word, for example dinfluencing (from directed influencing) for the concept but eventually dropped this idea. Probably the medicine would be worse than the disease.

${ }^{4}$ In systems theory the concept partsystem is the more general term $[14,15]$. Three types of partsystem can be derived from any original system: a subsystem takes some of the elements from the original system, an aspectsystem concentrates on some of the relations of the original system, and, finally, a phase-system takes only part of the original time slice into consideration.
}

processes, teaching, trying to convince, organizational improvement, learning, governing, and so on. This relativity matches reality much more than, for instance, outstanding managers, administrators, and consultants are aware; they all strive for some successful influence on what they feel responsible for, but nevertheless realize that the effect of this influence is limited in principle.

Thus the concept of control is a relative one because of (1) the explicit distinction between the intentions of control as direct influence and the effect of this influencing, (2) the recognition of the discrepancies between these two, and, finally, (3) the definition of an observed activity as control is an act of an observer trying to grasp some aspects of reality.

The abstract nature of this concept of control does not imply the acceptance of some discernable subsystem: the controller. To be more specific, the process of management cannot be equated with the activities of the manager. While in many situations managers do indeed dominate this process, in principle every organizational member participates in it. The control system of an organization, therefore, is not by definition located in a subsystem. It has to be found in a partsystem, ${ }^{4}$ the relation of which to the system as a whole varies greatly with the type of organization. This partsystem might be a subsystem that is identical to the managerial subsystem. In such a case of extrinsic control, control is exerted by some (authoritarian) manager(s). The partsystem might also be an aspectsystem, for instance, when separate groups have self-control. In this situation, there is not a separate control system as a subsystem, but the control is produced by a specific bundle of relations between the members of the group. This type of control is called intrinsic control. Control might also be an emergent property: control resulting from the interactions. In contrast with extrinsic control, intrinsic control refers to more democratic and more participative forms of decision-making in organizations, which may be explicitly designed but may also emerge out of a process of interaction. According to Weick [55, p. 8], such control is often disrupted by managerial meddling. Management intervenes in the mistaken belief that single individuals do the controlling, denying that control may be produced implicitly in causal circuits and 
interpersonal influence processes. Failure to acknowledge these forms of self-control, coupled with interventions that actively disrupt these self-regulating activities, are the occasions for much mismanagement in organizations.

In addition to this structural type of intrinsic control, we can distinguish an ideological type of intrinsic control $[12,17]$. A shared ideology may solve many problems of control by specifying broad, tacitly understood rules for appropriate action under unspecified contingencies. For instance, Mintzberg connects ideological control to his concept of the missionary organization [33] and the ideological strategy [34]. In both these concepts, control is produced by a shared ideology that offers organizational members an attractive identity as well as convincing interpretations of reality. Furthermore, there is no need for conscious actions taken by the controller. In connection with this, Adam Smith's invisible hand may indeed be considered as such an abstract intrinsic controller referring to a bundle of relations that form the market producing the coordination. To give a final example of the broadness of the concept, it also embraces several types of control Weick [57] distinguishes in his theory of "organization redesign as improvisation", such as "control by paradigm" and "control by enacted stability".

We wish to note that from the point of view of systems theory, intrinsic control is not necessarily superior to extrinsic control: this depends, among other things, upon the goals and circumstances. To take an organizational example of intrinsic control which can be judged negatively, one can imagine a situation in which organizational members are all prisoners of the bundle of relations that tie them together. In the same way, an organization can also be a mental prison [36] if the culture hampers seeing important changes in the market. Thus, in terms of control, culture is then an intrinsic controller that effectively controls the organization with respect to the preservation of ways of thinking that may become outdated over time.

Finally the abstract nature of control enables an individual, or individuals, to change roles between being the controller and being the

${ }^{s}$ We refrain from further analysing power relations, although this would be illustrative of the theory. The simple reason is that it would take such a large amount of space that the main ideas of looking at flexibility as dual control would be obscured. controlled, a fact which offers us a possibility to model the idea of dual or reciprocal control that we take as a fundamental key for analysing flexibility.

\section{MODELLING DUAL CONTROL: THE CONTROLLING ORGAN-TARGET SYSTEM GAME}

In order to take the idea of dual control further, we can use one of the main concepts of the system theory of control: the so-called controlling organ (CO) target system (TS) modelling game. The CO-TS game offers a framework to facilitate the analysis of organizational phenomena from several contrasting or even conflicting points of view. It is called a game because of the need to use the framework in a creative and playful way in order to provide this facilitation. This CO-TS game explicitly forces researchers and managers to look at organizations from a variety of angles, as networks of COs and TSs. As a general modelling rule of the game, whenever a partsystem, $\mathrm{A}$, is seen as a CO and another is seen as TS, B, being controlled, one must also model the reversed picture in which $\mathrm{B}$ is seen as the $\mathrm{CO}$ and $\mathrm{A}$ as the TS. This multiform framework avoids the mono-paradigmatic mechanistic and one-sided images of the organization, as, unfortunately, is often the case with approaches derived from traditional systems and control theory. For example, it is no longer beyond the scope of the systems theory of control to analyse power relations. As is well-known, most theories view power relations as dual relations: the power of $A$ over B and the power of B over A. Both have to be analysed, which is perfectly in accordance with the CO-TS game. ${ }^{5}$

To elucidate the multiple frames of this game for creative modelling, we should recall that the most fundamental decision in applying systems theory is the act of defining the system concerned, thus distinguishing it from its environment. The establishment of these system boundaries is inescapably associated with a certain cognitive point of view; i.e. a particular set of presuppositions and attitudes, a perspective, or a frame. According to Goguen and Varela [22], one may choose to focus one's attention (cognitive point of view, frame) either on the system's environment, taking the system's properties as given, or else on the 
internal constitution of the system. In other words, in line with the CO-TS game, we can think of an organization as a controlled system and as an autonomous system, thus capturing the idea of dual control that we believe to be central to flexibility. As we will see, this leads to understanding different types of flexibility.

\section{The organization as a controlled system}

Modelling the organization as a controlled or target system, the observer chooses to pay attention to the environment, treating the system as a simple entity with given properties and seeking the regularities of its interaction with the environment; i.e. the constraints on the behaviour of the system imposed by its environment (see Fig. 1). This leads naturally to the problem of controlling the behaviour of the system, as considered in the traditional systems theory sometimes called Cybernetics I.

Cybernetics I has been developed extensively as a result of the study of man-made systems (such as engineering and computer systems), whereas the insights derived from natural systems have remained by and large much less formally developed. From the viewpoint of Cybernetics I, organizations are seen as open systems, which is a result of attempts to make sense of such systems from the standpoint of an external observer. These open systems are in constant interaction with their environment, transforming inputs into outputs as a means of creating the conditions necessary for survival $[36$, p. 236]. Fluctuations in their environment are viewed as challenges to which the organization must respond. This is the classical idea of adapting. In this context, fluctuations are viewed as equilibrium-disturbing. Therefore, for a system to remain stable, it is believed that fluctuations must be minimized [46, p. 273].

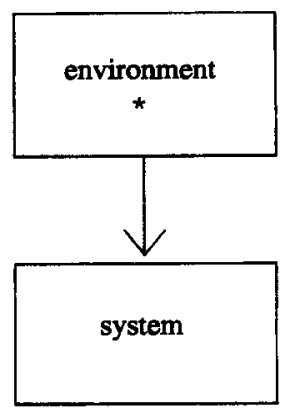

* = cognitive viewpoint or observer

$\downarrow=$ flow of signals and interactions

Fig. 1. The organization as a controlled system

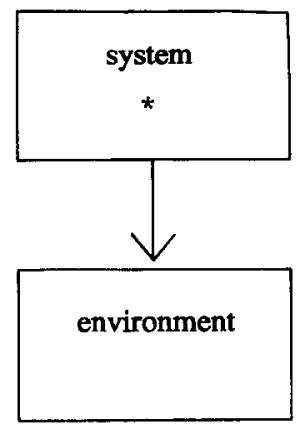

* - cognitive viewpoint or observer

$\uparrow$ - flow of signals and interactions

Fig. 2. The organization as an autonomous system.

\section{The organization as an autonomous system}

The CO-TS game demands that the reverse stand is also taken. An observer may choose to focus on the internal structure of the system, viewing the environment as background; e.g. as a source of perturbations of the system's autonomous behaviour (see Fig. 2). From this viewpoint, sometimes called Cybernetics II, the properties of the system emerge from the interactions of its components. In other words, environmental influences become perturbations which are compensated for through the underlying recursive interdependence of the system's components. This perspective of autonomous systems also challenges the validity of absolute distinctions between a system and its environment that underlie Cybernetics I.

The notion of autonomy is particularly important for natural systems, for example biological and social systems, and the lack of a well-developed theory of autonomous systems is felt to be a serious problem.

"An engineer designing an artefact will choose the inputs of interest to him for this application with some assurance that the choice will be adequate. But a biologist studying a cell is forced to acknowledge the autonomy of the cell; if the biologist's preferences for input and output variables do not match the cell's internal organization, the biologist's theory will not work" [22, p. 35].

Some researchers, however, in particular Maturana and Varela [32], offer a new perspective for understanding the logic through which living systems change, which is the foundation of Cybernetics II [36]. In this theory, all living systems are organizationally autonomous systems of interaction that make reference only to themselves. Maturana and Varela have coined the term autopoiesis to refer to this capacity for self-production through a closed system of relations. However, they 
developed their theory primarily as a new interpretation of biological phenomena, and they have strong reservations about applying it to the social world. Accordingly, the research literature concerning Cybernetics II suggests that there is a difference between naturally occurring autopoietic structures and those of social entities. Maturana and Varela therefore propose the use of 'autonomy' instead of 'autopoiesis' as the proper term for referring to the identity-preserving capability of 'social organizations'. ${ }^{6}$

Autonomous organizations strive to maintain their identity by subordinating all changes to the maintenance of their own organization as a given set of relations [32, pp. 80-81]. They do so by engaging in circular patterns of interaction whereby change in one element of the system is coupled with changes elsewhere, setting up continuous patterns of interaction that are always self-referential. This implies that a system's interaction with its environment is a reflection of and part of its own organization. Consequently, the system cannot enter into interactions that are not specified in the pattern of relations that define its organization. This so-called 'organizational closure' does not mean that an autonomous system is completely isolated in the sense that it has no environment, but that the relations with any environment are internally determined; its environment is really defined by, and in some sense is even a part of, itself.

If autonomous organizations are geared to maintaining their own identity, and if relations with the environment are internally determined, then systems can evolve and change only along with self-generated changes in identity. Therefore, in Cybernetics II, attention is drawn to system processes that try to maintain identity by ignoring or counteracting threatening fluctuations, and to the way variations can lead to the emergence of new modes of organization. Random changes can trigger circular interactions, the final consequences of which are determined by whether or not the current

${ }^{6}$ Furthermore, Morgan [36] remarks that, used as a metaphor, the theory of autopoiesis has intriguing implications for our understanding of organizations.

${ }^{7}$ Smith [46, p. 273] perfectly illustrates this concept of "order through fluctuation" by considering the predator-prey relationship of lynxes and rabbits. identity of the system will dampen the effects of the new disturbance through compensatory changes elsewhere, or whether a new configuration of relations will be allowed to emerge. In this context, fluctuation is viewed as a major vehicle for creating order, not for destroying it.? For instance, the theory of dissipative structures founded by Prigogine [39] suggests that nearequilibrium order is destroyed, whereas far-from-equilibrium order is maintained. According to Smith [46, p. 275], autonomous systems strive for resilience instead of stability. Resilience may be viewed as a measure of the persistence of a system and its ability to absorb change and disturbance and still maintain the same relationship with other entities within its environment. It is therefore possible to think of an autonomous system becoming more unstable as a result of large fluctuations, but knowing how to survive with these fluctuations makes for greater resilience in that many changes can be absorbed.

It will become clear later that this concept of an autonomous system in the sense of the autopoietic system leads to a different idea of flexibility than that arrived at through the concept of Cybernetics I.

\section{Organizations: controlled and autonomous systems}

In summary, organizations can be considered from different points of view, namely as controlled or as autonomous systems, each leading to different types of flexibility. It is not our intention to join these various perspectives together in a single theory. Goguen and Varela $[22$, p. 36] argue that these views are complementary, in the sense of contributing to a better understanding of natural systems.

The CO-TS game offers a frame within which these various preferred views on systems can be unified. Playing this game further we shall obtain a number of different CO-TS configurations on which to base our concept of flexibility. Therefore, the examination of the organization as a controlled and as an autonomous system will be undertaken. For each configuration, flexibility is defined in terms of properties of control. In particular, it is argued that organizational flexibility makes an organization uncontrollable from its environment and/or powerful in controlling its environment. 
In the following first round of this CO-TS game, we will deal more explicitly with organizational flexibility in relation to control. By further analysing the standard CO-TS model, we will clarify how flexibility contributes to control. This exercise helps us to define flexibility as a two-dimensional concept, namely as a function of the control capability of the management and of the controllability of the organization. When flexibility is seen as a management task the concern is with the quality of the control capacity of the management; when it is seen as an organizational design task the concern is with the controllability of the organization and the environment under different conditions.

Subsequently, in the second round of the $\mathrm{CO}-\mathrm{TS}$ game, these management and organizational design tasks will be examined further. This will finally result in a systematic and multidimensional picture of flexibility.

FLEXIBILITY FROM DIFFERENT CONTROL PERSPECTIVES: THE FIRST ROUND OF THE CO-TS GAME

In this first round we shall explore an organization as a controlled and as an autonomous system. Each configuration has different implications for flexibility.

\section{The organization as a controlled system}

To begin with, the organization is considered as a controlled system. In other words, the environment is defined as a controlling organ and the organization as a target system. As such, the organization must find a means of resisting the threat of incorporation by its immediate environment, and the consequent loss of distinctiveness $[35, \mathrm{p}$. 28]. If the environment can directly force the organization in a certain direction, regardless of the presence of organizational controls, the organization may become adrift. ${ }^{8}$ Hence, the organization seeks a degree of freedom from environmental

${ }^{8}$ In his typology of various organizational strategies, Mintzberg [34, p. 268] has called this an imposed strategy. These strategies originate in the environment. The environment dictates patterns in actions either through direct imposition or through implicitly preempting organizational choice. influence $[6,11,18]$. As a result, the organization adapts in such a way that it remains essentially the same, despite changes in the environment [17].

In this configuration, flexibility stands for insensibility to control from the environment. Or, in more specific terms, high flexibility corresponds with low controllability from the environment. Low controllability implies that the target system, in this case the organization, cannot be directed by the environment towards the desired set of states. The organization is not a puppet in the hands of its environment. One might think of it as being elusive. We have an internal type of flexibility here, which is often activated in response to environmental change; i.e. whenever the environment exercises control over the organization it is able to adapt in such a way that this control does not succeed: the environment loses hold.

\section{The organization as an autonomous system}

Using the turn-around rule of the game, we shall now consider the organization as an autonomous system; i.e. the organization is defined as a controlling organ and the environment as a target system. In this configuration the organization tries to influence the environment. In order to produce directed influence upon its environment, the organization needs to possess some dominance. Flexibility then means the ability to successfully control the environment, or, in common language, to manipulate the environment. In more specific terms, high flexibility corresponds with an extensive control capacity of the organization with respect to the environment. We have an external type of flexibility here which can be passive or active: with or without a trigger from the environment, the organization is able to take successful initiatives in directing the environment towards states that are favourable for the organization.

Table 1. Organizational fiexibility considered from different configurations of control

\begin{tabular}{lc}
\hline \multicolumn{2}{c}{ Perspective of control: the organization as } \\
\hline \multicolumn{1}{c}{ Controlled system } & Autonomous system \\
\hline $\begin{array}{l}\text { Seeking autonomy } \\
\text { Adapting by remaining partly itself }\end{array}$ & $\begin{array}{c}\text { Seeking dominance } \\
\text { Producing directed influence } \\
\text { upon its environment }\end{array}$ \\
Passive or active & External flexibility \\
\hline
\end{tabular}




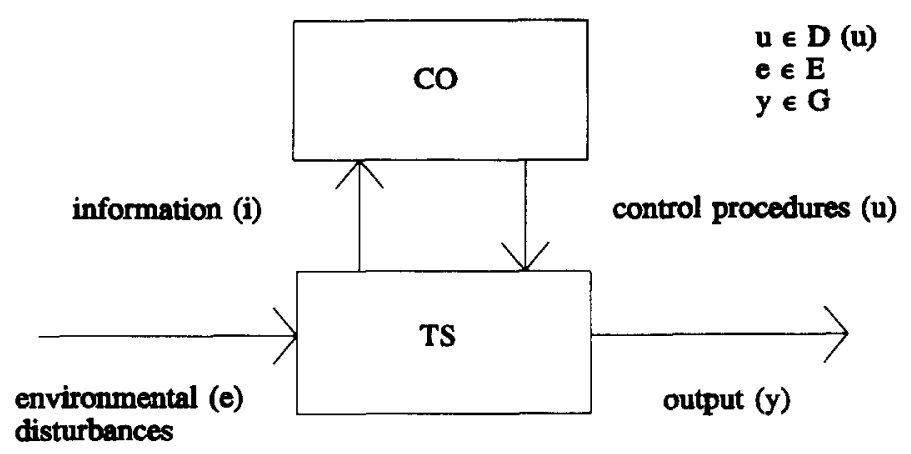

Fig. 3. A standard CO-TS model.

The two configurations explained above and their implications for organizational flexibility are summarized in Table 1.

So far we have seen that the use of a control perspective results very naturally in a distinction between internal and external flexibility (producing changes in the organization or in the environment). Subsequently, we might argue that each of these types can be further subdivided into passive and active types (behaving with or without a trigger). As a result, four types of flexibility emerge:

-passive internal: ability to generate effective internal changes in response to environmental changes (e.g. changing the existing structure itself in a simple way when this proves to be necessary);

-active internal: ability to generate internal changes that are favourable without being first triggered by the environment (e.g. investments in underused assets such as increasing the liquidity of assets, using general-purpose facilities and equipment, maintaining extra $R \& D$ capability or using inventories as a buffering mechanism);

-passive external: ability to restore external changes (e.g. developing a product-market posture which is sufficiently diversified to minimize the effect of a change in demand);

-active external: ability of the organization's own initiative to control the environment in an effective way (e.g. putting the firm into areas in which it can benefit from likely breakthroughs).

${ }^{9}$ For a further elaboration of various types of flexibility on the basis of the systems theory of control, we refer to Volberda [52, pp. 117-128].
The above distinctions of types of flexibility can indeed be found in the relevant liferature. For instance, passive and active external flexibility correspond with Ansoff's [7] defensive and offensive external flexibility. Moreover, passive and active internal flexibility are considered by Reichwald and Behrbohm [42] in terms of reactive and anticipative flexibility. However, the systems theory of control clearly illustrates that both internal and external flexibility can be either passive or active. This eradicates a great deal of the ambiguity in the literature concerning active and passive flexibility. A further elaboration of types of flexibility might be useful here but at the same time might result in complexity that is unnecessary at this point and that may even obscure the argument. ${ }^{9}$

In the remainder of this article, we will limit ourselves to a further exploration of some aspects of the two configurations that have been introduced here, which will gradually result in an integral concept of flexibility. In particular, we will explore the two questions previously formulated, namely "What makes an organization uncontrollable from the environment?" and "What makes an organization powerful in controlling its environment?" To answer these questions we need a better understanding of how flexibility contributes to solving problems of control.

\section{FLEXIBILITY IN TERMS OF PROPERTIES OF CONTROL}

A problem of control can be represented as a standard CO-TS model; i.e. an organization consisting of a configuration of a $\mathrm{CO}$ and a TS which is positioned in a certain environment. In this configuration, as portrayed in Fig. 3, the solvability of the control problem is determined 
by the control procedures, the controllability of the TS, and the control capability of the CO.

\section{A specification of the control problem}

In order to solve this problem of control we must first start with a specification of the control problem [15, p. 118]. This involves:

(i) a specification of the system to be controlled (TS), which requires some modelling decisions regarding the system in question, such as the establishment of system boundaries, the choice of the appropriate level(s) of aggregation, or the partsystem to be considered;

(ii) a specification of the goals strived for, or at least some evaluation mechanism (e.g. suppose that only certain outputs $y$ within a desired class $\mathrm{G}$ are acceptable);

(iii) a specification of the environmental circumstances (e.g. suppose that the environmental disturbances $e$ will be limited to influences within a class $E$ ).

On the basis of the specification of this problem of control, it is clear that flexibility is always relative to the goal strived for and the environmental circumstances. One might generalize this idea by defining flexibility as relative to a class of goals $(\mathrm{G})$ and a class of environments (E). A system is flexible if it can attain any of the class of goals in any of the environments from the class $\mathrm{E}$. The relativity, however, remains. This confirms the intuitive feeling that absolute flexibility is nonsense. According to Smith [45, p. 321], this notion implies that the meaning of flexibility does not lie within the behaviour itself, for otherwise its meaning would remain constant across time, situations, and levels of analysis. As was illustrated in the above CO-TS game, the meaning of flexibility changes depending on the context and on whose system of attribution we are talking about. Organizational flexibility, therefore, is not a characteristic of an organization itself. Rather, in line with Smith's argument, it is a characteristic of the relationship between an organization and its environment, even though it may be expressed or made visible in the actual behaviour of an organization. From a practical stand, the relativity of flexibility leads to a very natural linkage between flexibility, organizational strategy and environment, which is indeed recognized in some definitions of flexibility (e.g. $[2,28,40,41])$.
Following on from this specification, we have to estimate the solvability of the problem of control. We should restate that, regarding the solution of this problem of control, both the controllability of the TS and the control capability of the $\mathrm{CO}$ must be considered.

\section{Controllability of the target system}

Controllability is the possibility of reaching the goals $G$ with the TS, irrespective of disturbances $e$ from the environment $\mathrm{E}$. Therefore, a more precise definition calls a system TS $[\mathrm{G}, \mathrm{E}]$ controllable if for every $e$ from $\mathrm{E}$ there is a control procedure $u$ from the available set $\mathrm{D}(\mathrm{u})$ so that output $y$ belongs to the desired set $G$. In further refinements time is taken into account. Controllability, then, is concerned with the possibility of directing the TS from some arbitrary state to a desired state within some time period $T$ irrespective of environmental disturbances. We then call a TS $[\mathrm{G}, \mathrm{E}, \mathrm{T}]$ controllable. Even more precise definitions take into account a specified set of beginning states $\mathrm{S}$ from which a desired state can be reached. This leads to the most complex definition: a TS is called [G,E,S,T] controllable if, for every beginning state $s$ from $\mathrm{S}$ and irrespective of disturbances $e$ from $E$ within time $T$, the goal $G$ can be reached.

The link with organizational flexibility is quite simple. Roughly, flexibility increases with the increase of $E, G$ or $S$ and with decreasing $T$. The result of this analysis corresponds with common ideas about flexibility that have now been more systematically generated. An organization is more flexible if it can reach its goals in a larger set of environments. It can, so to speak, survive in different environments. It is more flexible if it can realize several goals from a larger set, if it is able to reach its goals starting from a larger set of initial states, and, finally, if it reaches its goals sooner.

\section{Control capability of the controlling organ}

Aside from the controllability, the other factor determining the effect of control is of course the control capability of the controlling organ $\mathrm{CO}$. The control capability is the ability of a $\mathrm{CO}$ to get the maximum of a TS; i.e. really using a TS to its full controllability potential. It is important to recall that we defined controllability as an absolute property of a TS. 
Controllability is thus independent of the properties of the controller. It may be helpful to draw an analogy with a car and a driver. The controllability of the car consists of properties such as road-holding and manoeuvrability. These are independent of the properties of the driver. In other words, they are defined with respect to a perfect driver. By contrast, control capability is a property of a CO. Similarly, one can speak of good drivers without referring to a specific car. Looking at the control capability more fundamentally, one realizes that theoretically it would be more correct to define control capability in relation to a class of TS. However, in view of the aim of this article, we will abandon that exercise. Although in any real situation $\mathrm{CO}$ and $\mathrm{TS}$ are indeed interrelated, the analytical distinction is essential.

When the control capability fails, one does not get the maximum from a certain TS. The controllability refers to the absolute boundary of some TS which can only be realized by a CO with sufficient control capacity. When there is a shortage of control capacity, this may result, for instance, in not reaching the goal for some elements from $\mathrm{E}$ or $\mathrm{S}$, and also in taking a longer required time to reach the goal.

The theory of control gives us some insights into the question of what determines control capability. Among other things, these are the requirements for effective control $[15,16]$, in particular those that have not yet been mentioned in the definition of controllability, namely:

(iv) the quality of the model; the model must incorporate the relationships between environmental disturbances, control actions and the resulting outputs. Or, in a mathematical formulation, the controller must search for an adequate function $f$ in $y=f(e, u)$. The higher the quality, the better the effect of a control procedure can be predicted, which facilitates the search for an effective measure;

(v) the set of control procedures; the set $D(u)$ of the controller must be larger than, or equal to, the set of disturbances $e$ within $\mathrm{E}$. This is Ashby's [8] law of requisite variety;

(vi) the capacity of dealing with information must be large enough; according to Conant [13], a student of Ashby, some of this capacity is wasted on noise $\left(I_{n}\right)$, on blocking irrelevant data $\left(I_{\mathrm{b}}\right)$, and on coordination $\left(I_{\mathrm{c}}\right)$. In a case where there are many interfaces within the $\mathrm{CO}$ together with a large amount of incoming irrelevant data, the net information-handling capacity $\left(I_{\text {net }}\right)$ is strongly reduced; i.e. $I_{\text {net }}=I_{\text {tot }}-I_{n}-I_{b}-I_{c} . \quad I_{\text {net }}$ is the maximum capacity the controller can deal with.

We should repeat that these requirements for effective control are necessary but not sufficient: a lack of controllability of the TS cannot be overcome by better control capabilities. Moreover, the requirements do not in general counterbalance each other. For instance, a shortage in capacity for handling information cannot be compensated by an overkill in procedures for control, and vice versa. Still, there are some exceptions if one incorporates time. For instance, the predictive capabilities of the $\mathrm{CO}$ (which are determined by the quality of the model, the capacity of sensing information from the environment, and the information-handling capacity) might enable it to adopt successful procedures early enough, whilst, if left any later, the set of control procedures would have been insufficient. This corresponds to the old Dutch proverb "If one is not strong one has to be smart" or "Necessity is the mother of invention". Incidentally, the fact that many of the abstract conceptual issues discussed above are found in common wisdom, especially in proverbs, ensures that we have not lost contact with reality.

\section{Flexibility and control}

The relations between control and flexibility following from this exploration of the $\mathrm{CO}-\mathrm{TS}$ game are simple: flexibility corresponds with high controllability and large or at least sufficient control capability. Therefore, the resulting flexibility of a CO-TS configuration is the minimum of controllability and control capability. In terms of the analogy of the car: the flexibility of a car-driver combination is the minimum of the steering capacities of the driver and the control properties of the car. Aspects such as the state of the road and the weather are also present; they are some of the environmental circumstances. So flexibility is a function of two sets of variables and not, as is sometimes suggested, a function of one of the two. In terms of management and organization, flexibility is a function of the control capability of the management and the changeability of the organization. They cannot be counterbalanced: e.g. a too restricted changeability of the 


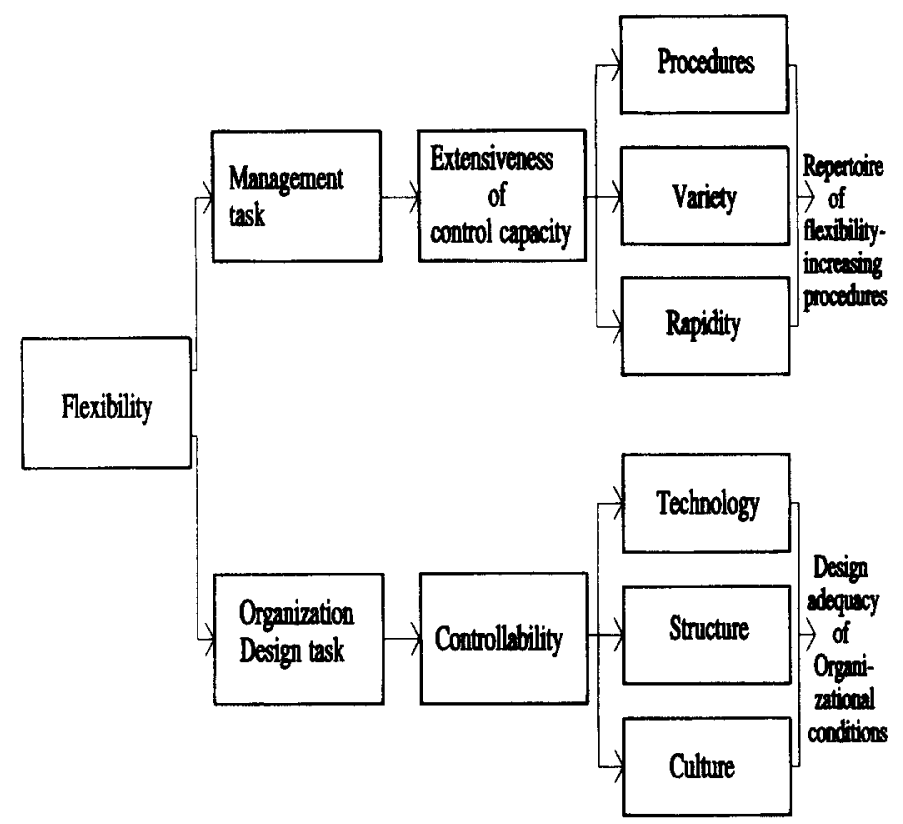

Fig. 4. Organizational flexibility and the associated management and organization tasks

organization cannot be compensated by the extra effort of the management.

\section{A MULTIDIMENSIONAL DEFINITION OF ORGANIZATIONAL FLEXIBILITY}

As a result of the above-mentioned considerations, we found that flexibility has to be treated as a two-dimensional concept; i.e. there are two distinct and equally important challenges to be met if flexibility is to succeed (see Fig. 4). First, flexibility is seen as a management task. In this respect, the concern is with the quality of the 'control capacity' or the competence of the management. Second, flexibility is seen as an organization design task. The concern here is with the 'controllability' of the organization and the environment which involves the creation of the right organizational conditions to foster flexibility: is it possible to implement different types of flexibility within the organizational context? These two dimensions result in the following definition [52-54]:

Flexibility is the degree to which an organization possesses a variety of actual and potential procedures, and the rapidity by which it can

${ }^{10}$ Other requirements are interesting too: e.g. the requirement of a model tells us that the managerial dimension of flexibility is bound by the quality of the model management has of its organization and its environment. In short, no flexibility without real insight. implement these procedures, in order to increase the control capability of the management and improve the controllability of the organization and the environment.

This seemingly complex definition is explained below.

\section{The management task}

As a continuous management task, flexibility is concerned with the creation or promotion of the management's control capacity, especially in situations of unexpected disturbance. This management task must be fulfilled in order to prevent a threatening uncontrollability of the organization. Restraining ourselves to some of the requirements for effective control ${ }^{10}$ we take as core components of this management task:

(i) the existence of actual and potential procedures of management; not only the actual range of procedures is important, but also the range of potential procedures, which have not yet been activated. Actual procedures have already been allocated to a real flexibility-need [42]. The possible emergence of opportunities or threats, however, requires management to have some potential procedures to rely upon as an insurance against risk [43].

(ii) the variety of procedures of management; Ashby [8] demonstrated that the required variety of procedures activated by the manage- 

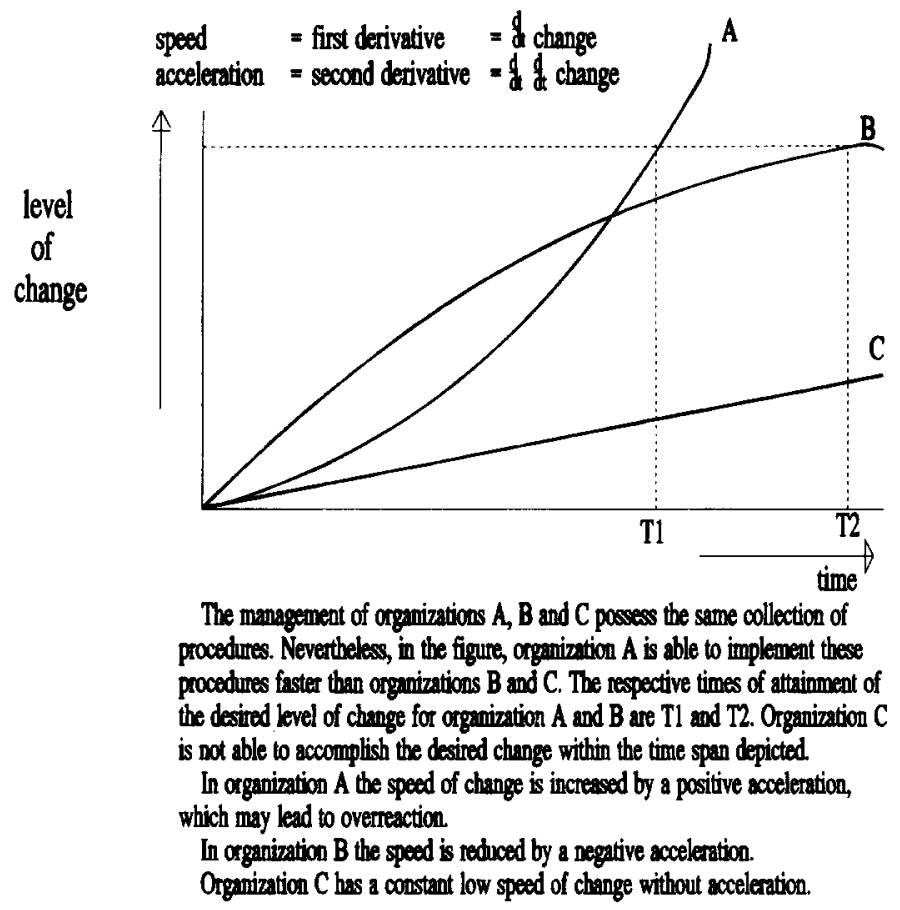

Fig. 5. Flexibility and response time.

ment within the organization must be minimally equal to the variety of disturbances within the environment. This is the 'Law of Requisite Variety': variety in the environment can only be absorbed by variety in the organization. The variety of procedures of the organization has to be equated with the extent of disorder within the environment which the management wishes to control. In many organizations, the management reduces the perceptible variety in the environment to below the threshold level of required response by using various reduction mechanisms, such as standardization, formalization and complex planning systems [9]. Consequently, the repertoire of control procedures and the controllability of the organization are strongly diminished. In a turbulent environment, however, the management has to possess a large variety of procedures; in other words, an extensive collection of flexibilityincreasing procedures in order to control the organization and its environment. This large variety of procedures of management can be in terms of either the quantity (the number of procedures) or the quality of the procedures (such as one-off versus durable flexibility-increasing procedures). For instance, the training of multi-skilled personnel results in a more durable mode of flexibility, while the contracting out of certain peripheral activities or "hire and fire' employment practices tend to result in a one-off improvement in flexibility. One-off flexibility-increasing procedures lead to a reduction of the potential for use once allocated, whilst durable flexibility-increasing procedures are not restricted in use.

(iii) the rapidity by which management can implement its procedures; management may possess the right procedures, but this does not necessarily mean that it can implement these procedures in time (see Fig. 5). Flexibility is not a static condition, but a dynamic process. Time is an essential factor of organizational flexibility. However, a short comment must be given on the appreciation of speed in popular management beliefs. For instance, in a popular article in Fortune, Dumaine [19] describes how management can succeed through speed. Too short a reaction time, however, may lead to overreaction and excessive information searching and finally result in chaos. From the theory of control we know that the Controlling Organ should take account of the lead time of the Target System: a feedback that is too fast leads to instability and eventually to the explosion of the system. Therefore, the management some- 
times has to act prudently instead of decisively in that it must wait until the impact has reached a certain threshold level (increase of retardation time) or slowly and continuously implement change (increase of response time). In the case of prudent management, the mean acceleration of the process of change is reduced. Thus, rapidity of implementation is a function of the required time and the acceleration of the process of change.

\section{The organization design task}

Nonetheless, there are some limitations to the control capability of the management. The management's ability to initiate flexibility-increasing procedures is dependent upon certain organizational conditions, such as the organization's technology, structure and culture. These determine the volume and composition of the collection of flexibility-increasing procedures.

This is in line with De Leeuw's [15] "Law of Managerial Busyness", which demonstrates a decreasing control performance as soon as control efforts are increased above a certain critical level. As is depicted in Fig. 6, the relation between the use of the control capability of the management and the controllability of the organization roughly resembles a parabola. In accordance with this law, when the management tries to increase the collection of flexibility-increasing procedures beyond its limits, the result is that the controllability of the organization diminishes further and further.

The controllability or the absolute boundary of potential flexibility inherent in an existing organization depends on the design adequacy of the organization [59]. Design adequacy is a measure of the probability that the organization's technology, structure and culture are changeable within the time window required by the repertoire of flexibility-increasing procedures. Thus, the controllability of the organization determines the boundary conditions imposed on the collection of flexibility-increasing procedures. Therefore, an organization that has a surplus of flexibilityincreasing procedures, that are all activated by the management, will experience chaos. Organizations sometimes have to vary their boundary of potential flexibility by changing the design parameters of the technology, structure or culture.

On the one hand, this implies a possibility of enlarging flexibility by changing (i.e. widening) the organizational conditions. This corresponds with Galbraith's [20] design strategy of creating organizational slack. The extreme consequence of this strategy for improving flexibility is an organization with organizational conditions so loose that it becomes nonexistent: all possible states are considered satisfactory. We then have flexibility by the grace of the absence of an identity or mission stemming from the organizational culture, a structure characteristic of the organization, or a distinct technology. It may be clear that this is no flexibility at all. No control action is needed any more because the organization is happy, irrespective of the present state. Compare the person that holds all opinions, even conflicting ones, depending on the direction of environmental pressures. One then concludes that this person has no opinion at all. On the other hand, an organization is flexible when the minimum of controllability

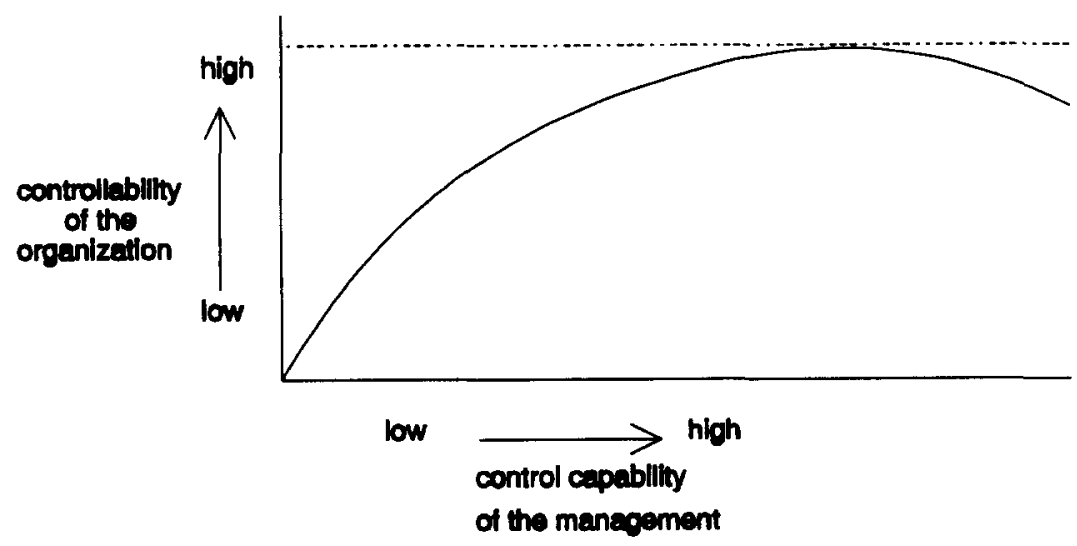

Fig. 6. Law of managerial business. 
and control capability is high, especially in the case of tight organizational conditions. In the latter case, a small set of desired states can be reached notwithstanding disturbances. The essence is that the change is restricted: not everything is changeable.

This amounts to the insight that flexibility is linked to tenacity with respect to organizational conditions as well as to their adaptability [50]. The concept of flexibility is not only opposite to but even impossible without preservation. This apparent contradiction is very important for understanding flexibility. The notion of preservation leads to the consideration of the second dimension of flexibility, namely the organization design task [21]. This task involves creating the appropriate organizational conditions necessary to effectively realize certain flexibility-increasing procedures. They thereby guarantee 'preservation' for the organization and some 'stability' for its participants. These frequently neglected aspects are indispensable elements for the realization of flexibility [51]. Just as there can be no differentiation without integration [29], similarly, there can be no flexibility without some stability or preservation [5]. Stability provides certainty for organizational members and preservation facilitates controllability of the organization. Certainty refers to situations in which organizational participants have enough possibilities of preserving their identity. If there are not enough possibilities, participants will lose their identity. Both controllability of the organization and certainty for organizational participants define the limits of various types of flexibility.

\section{The paradox of flexibility}

A controllăble form of flexibility requires some preservation regarding organizational conditions; at the same this preservation defines the boundaries and limits of the collection of flexibility-increasing procedures. Thus, our two-dimensional conception of flexibility creates a paradox: an organization must possess some procedures which enhance its flexibility in order to avoid becoming rigid, but it must also be anchored in some way in order to avoid chaos. Rather than accepting the dichotomy of preservation and change, this paradox implies that organizational flexibility incorporates both change and preservation. Consequently, management has to deal with a constructive tension
[25] between that which must be changed and that which it is necessary to preserve; a tension, for example, between the need for managers to question and challenge versus the preservation of core values and organizational mission; between the need for new ideas and directions and the need for continuity and preservation of core technologies [24]. Or, as Ulrich and Lake [49, p. 245] formulate it, the flexible organization asks for a willingness to shift, flex and change, and at the same time for an unconditional commitment, concern, and loyalty to the organization. As we have stated above, this preservation or anchoring can be a result of various dimensions of the organizational culture, the organizational structure, or the operational technology.

\section{RECONSIDERING THE MANAGEMENT AND ORGANIZATION DESIGN TASKS OF FLEXIBILITY: THE SECOND ROUND OF THE CO-TS GAME}

The systems theory of control has resulted in a systematic and multidimensional definition of organizational flexibility. In the second round of the CO-TS game we will further elaborate the two configurations as discussed above. In particular, their management and organization design tasks concerning flexibility are considered. It is our argument that such explorations contribute to insights which support our definition of flexibility.

\section{The management and organization design tasks within a controlled organization for improving adaptability}

In the case of a controlled organization (see Fig. 7), the organization is considered as a system (TS) steered by its environment (CO). In the first half of the game, we described flexibility in this context as being insensitive to environmental influences. A further examination teaches us that we have two alternatives: insensitivity together with a total absence of control activity within the organization, and insensitivity that is the result of effective control reactions for compensating environmental influences.

The first alternative might certainly be useful for organizations. One could think of a monopolist producing goods or services that are indispensable to the environment. In such a case the organization is unassailable because of the 


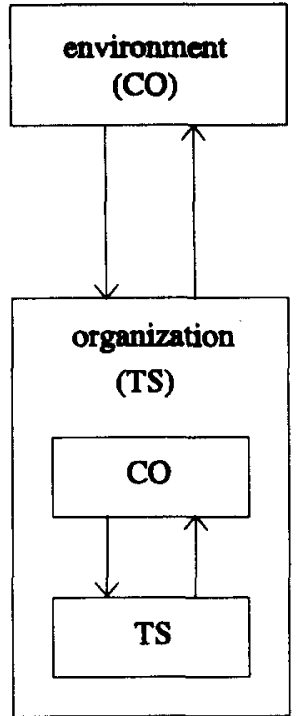

Fig. 7. The organization as a controlled system.

low control capability of the environment. Nevertheless, it does not seem to be natural to speak of flexibility in this case. The second alternative, however, does correspond with flexibility. In this case, disturbances are fought against effectively by alert adaptations (or, more broadly, changes) without the organization losing its distinctiveness. Consequently, the organization creates a degree of autonomy from environmental impacts by adapting in such a way that it largely remains itself. In order to examine the management and organization design tasks for this configuration we have to study the organization at a lower level of aggregation. This lowering of the level of aggregation is also called reticulation in the systems theory of control. As depicted in Fig. 7, reticulation of the organization as a TS results in a configuration of a $\mathrm{CO}$ and a TS in an environment. Using the insights from the systems theory of control as developed above, we may conclude that insensitivity to environmental disturbances is produced by high controllability of the TS (in the organization; mind the reticulation!) and sufficient control capability of the $\mathrm{CO}$.

In this context, the management task involves increasing the capacity to adapt to changes in the environment. Management can improve this capacity by activating flexibility-increasing procedures directed at change within the organization itself. This internal type of flexibility goes in tandem with changes in routines, structures and even goals of the organization. Nonetheless, management will only allow local or peripheral adaptations and will try as long as possible to retain a handful of central values. In other words, management must have sufficient competencies in terms of tenacity and willingness to adapt goals.

In order to assure that the environment cannot prevent the organization from going its way (it has a low controllability from the environment), the organization must be perfectly controllable for the management. The organization design task therefore involves the creation of organizational conditions which enhance the above described adaptability and preserve the core identity of the organization. From the theory of complexity, Simon's [44] concept of the viable system is immediately applicable. These organizations have neardecomposability properties and are arranged in a hierarchical way. The hierarchical structure implies that such organizations consist of subsystems which in turn consist of sub-subsystems, and so on. Near-decomposability means that the relations within the subsystems are stronger than those between the subsystems. The short-term behaviour of such subsystems is determined by the stronger internal relations, while the long-term behaviour of the system as a whole is mainly determined by the weaker interrelations between the subsystems. The hierarchical composition of relatively autonomous subsystems, or, in Weick's terms [56], loosely-coupled systems, possesses some unique properties of stability. In contrast with an unstable equilibrium, which is destroyed by every disturbance, Ashby [8] distinguishes three types of stability:

(i) monostability, which refers to an organization in which a certain position can be maintained in spite of certain disturbances; as long as the disturbances are not too large, this position can be restored. In other words, the organization is capable of keeping the essential variables within certain boundaries (homeostatic);

(ii) ultrastability, which stands for a higher form of stability; ultrastable organizations can reach totally different positions of equilibrium whenever circumstances change radically;

(iii) multistability, which refers to a characteristic enabling an organization to adapt locally in 
an ultrastable way to simultaneous and different disturbances.

Applying Ashby's stability concepts, we can subscribe ultra-stability properties to the subsystems in such a way that the system as a whole is multi-stable [17]. The last type of stability, which includes the first two, enables the organization to realize effective independent reactions which keep disturbances local (adaptability). It reduces the necessity for large-scale change but also makes it more difficult to achieve change when it is needed (preservation). This means that, in organizations designed according to these principles, disturbances are likely to be localized in their effects, with the activities of a system as a whole reflecting a great degree of persistence and stability in the face of changing circumstances.

Thus, in the case of a controlled system, the management task involves the activation of internal flexibility. Consequently, the environment has no hand in these organizations, not because they do not undergo influences but because they have great capacities to adapt. Furthermore, the organization design task involves creating a hierarchical composition of subsystems to facilitate the organization's adaptability, and also to preserve its core identity.

One more aspect has to be mentioned here, namely the fact that flexibility is related to various levels of aggregation [28]. From the above configuration, it is clear that flexibility at the level of the subsystems is needed to manage their internal relations. Consequently, the system as a whole is highly insensitive to local environmental disturbances. On the other hand, the system also needs another type of flexibility with respect to the weak interrelations between the subsystems. This latter type of flexibility is often centralized and involves the preservation of a handful of central values and the adaptation of peripheral values.

The management and organization design task within an autonomous organization for improving dominance

In the case of an autonomous organization (see Fig. 8), the organization is thought of as an organ (CO) that controls its environment (TS). It is seen as playing an active role in constructing its environment along with its own identity. It dominates the environment in order to preserve its identity. But some identities are likely to be more robust and enduring than others. As organizations assert their identities, they can initiate major transformations in their environment. Consequently, they can create the conditions that will allow them to evolve along with the environment or set the basis for their own destruction.

As portrayed in Fig. 8, domination over the environment requires high controllability of the environment and sufficient control capability of the organization. In this context, the management task involves enhancing the capacity to influence the environment. The management can increase this capacity by activating the flexibility-increasing procedures directed at change in the environment. This external type of flexibility sometimes goes together with changes in structures or goals of the environment. For instance, horizontal mergers and acquisitions reflect managerial attempts to control competitive uncertainties through adjusting industrial structure $[37,38]$. Vertical integration as an attempt to control input or demand uncertainties is also an example of external structural change [37, 58]. Finally, influencing consumers through advertising and promotions may even lead to changes in the goals of the environment [31].

In order to enable the organization to dominate its environment, this environment must be perfectly controllable from the organization. Of course, this is seldom the case. The organization design task therefore involves the organizing of the environment in such a way that it can be dominated by the organization. Just as we had to decompose the organization in the case of a controlled organization, in this

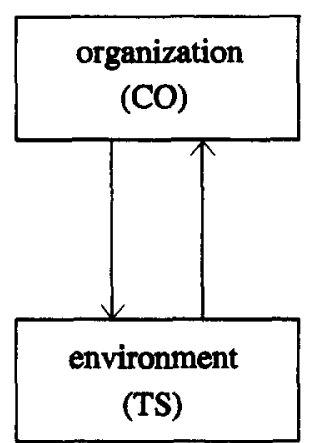

Fig. 8. The organization as an autonomous system. 
configuration we have to decompose the environment into relatively autonomous submarkets. In the literature of strategic marketing, we find many segmentation strategies aimed at successfully accomplishing such a decomposition (cf. $[1$, p. 51]).

Furthermore, by projecting itself onto its environment and thereby organizing its environment, an organization sets the basis for acting in relation to that environment in a way that actually allows it to produce itself; in other words, the self-referential capabilities of autonomous systems enable them to engage in various structural relationships with their environment. In Cybernetics II this is called structural coupling. These networks of relations enable the organization to constantly recreate the conditions necessary to sustain it. These relationships preserve the organization's identity ${ }^{11}$. While preservation of an identity is fundamental for the autonomous organization, there are different ways in which closure in relation to the environment can be achieved. In this context, we must keep in mind that the environment is not an independent domain, but a part of the organization because it is part of its domain of essential interaction. As Maruyama [30] expresses it, there is no longer room for parasitic relationships, where what one gains is another's loss, nor for antibiotic relations, where one, out of self-interest, actively harms the other. Instead, the organization has to create conditions that will allow it to evolve along with the environment. Organizations that only try to preserve their own fixed and narrowly defined identity may end up trying to sustain unrealistic identities, or ultimately destroying the contexts of which they are a part [36, p. 245]. An appreciation of the system's interdependence with suppliers, markets, labour force and other entities facilitates more symbiotic relationships. For example, one might think of long-term contractual agreements with suppliers or buyers, alliances or joint ventures, franchising agreements or technology licensing agreements.

"Smith [46, pp. 287-288] distinguishes three patterns of interaction, namely (1) communication, in which total autonomy of the entities is maintained, (2) fusion, in which autonomy of the entities is surrendered entirely so that the superordinate system will be maximally autonomous, and (3) symbiosis, in which each entity loses some autonomy in order to create an autonomous superordinate system.
Thus, in the case of an autonomous system, the management task involves the activation of external flexibility. Consequently, the organization can dominate the environment by producing directed influence. For this management task to succeed, the organization design task requires decomposition of the environment, and facilitating structural coupling with that environment in such a way that the organization's identity can be preserved and evolve along with that environment.

From the above configuration, it is once again clear that flexibility is related to various levels of aggregation. For instance, external flexibility is required to manage individual markets, but a higher level of external flexibility is required to manage the weak interrelations between markets. The former type of external flexibility is often decentralized, while the latter is often centralized within the organization.

\section{CONCLUSION}

In this article, we have sought to improve understanding of what the word flexibility might mean when applied to an organization. The notion of control was explored and led us to the idea of dual control, in which the organization is viewed as a controlled system and as an autonomous system. In the one case, flexibility is the organization being controlled by the environment, in the other the organization controls the environment. Four types of flexibility were identified, internal and external, each passive or active. Our discussion of the implications of these insights for organizational effectiveness and viability lead us to our multidimensional definition of organizational flexibility.

The management and organization design tasks, if flexibility is desired, were developed for different control configurations of the organization in relation to its environment. Of course, we can carry out the same exercise at higher or lower levels of aggregation, for instance, considering a subsystem in relation to the whole system with respect to flexibility. Still, it is true for every level of consideration, that a system's flexibility requires both adaptability and dominance if the system's identity is to be preserved and allowed to evolve along with that of the wider system. The paradoxical conclusion is that flexibility must not be seen as the opposite 
of stability but as a requirement of a higher order of stability: no flexibility without preservation.

\section{REFERENCES}

1. Aaker DA (1984) Strategic Market Management. Wiley, New York.

2. Aaker DA and Mascarenhas B (1984) The need for strategic flexibility. J. Busin. Strat. 5, No. 2, 74-82.

3. Ackoff RL and Emery, FE (1972) On Purposeful Systems. Aldine, Chicago.

4. Ackoff RL (1981) Creating the Corporate Future. Wiley, New York.

5. Adler PS (1988) Managing fiexible automation. Calif. Mgmt Rev. Spring, 34-56.

6. Aharoni Y, Maimon Z and Segev E (1978) Performance and autonomy in organizations: determining dominant environmental components. Mgmt Sci. 24, 949-959.

7. Ansoff HI (1965) Corporate Strategy. McGraw-Hill, New York.

8. Ashby WR (1964) An Introduction to Cybernetics. Methuen, London.

9. Beer S (1985) Diagnosing the System. Wiley, New York.

10. Burrell G and Morgan G (1979) Sociological Paradigms and Organizational analysis. Heineman, London.

11. Burton RM (1984) Variety in strategic planning: an alternative to the problem solving approach. Columbia J. Busin. Winter, 92-98.

12. Camerer $C$ and Vepsalainen A (1988) The economic efficiency of corporate culture. Strategic Mgmt J. 9, 115-126.

13. Conant $R$ (1991) Laws of information which govern systems. In Facets of Systems Science (Edited by Klir GJ), pp. 419-448. Plenum Press, New York.

14. De Leeuw ACJ (1980) Organizations, systems, wholes and parts: variations on a theme in organization theory. Method. Sci. 13, No. 4.

15. De Leeuw ACJ (1982) Organisaties: Management, Analyse, Ontwerp en Verandering. Van Gorcum, Assen/Maastricht.

16. De Leeuw ACJ (1982) The control paradigm: an integrating systems concept in organization theory. In Understanding and Managing Strategic Change (Edited by Ansoff I et al.), pp. 194-217. North Holland, Amsterdam.

17. De Leeuw ACJ (1993) Organizations: intelligent systems of expertise. In The Expert Sign: Semiotics of Culture (Edited by Slikkerveer LJ et al.), pp. 83-102. DSWO Press, Leiden.

18. Dill WR (1958) Environments as an infiuence on managerial autonomy. Admin. Sci. Q. 2, 409-443.

19. Dumaine B (1989) How managers can succeed through speed. Fortune February, 54-59.

20. Galbraith J (1973) Designing Complex Organizations. Addison-Wesley, Reading, Mass.

21. Gazendam HWM, Rutges AC and Volberda HW (1993) Analysis of organizations using multiple metaphors. In The Expert Sign: Semiotics of Culture (Edited by Slikkerveer LJ et al.), pp. 245-275. DSWO Press, Leiden.

22. Goguen JA and Varela FJ (1979) System and distinctions: duality and complementarity. Int. J. gen. Syst. 5, 31-43.

23. Jackson MC (1991) Systems Methodology for the Management Sciences. Plenum Press, New York.

24. Johnson G (1988) Rethinking incrementalism. Strategic Mgmt J. 9, 75-91.
25. Kanter RM (1983) The Change Masters. Simon \& Schuster, New York.

26. Kickert WJM (1982) Het toverwoord flexibiliteit. Bestuurswetenschappen Oct./Nov., 476-492.

27. Kickert WJM and Van Gigh JP (1987) A metasystem approach to organizational decision making. In Decision Making About Decision Making (Edited by Van Gigh JP), pp. 476-492. Abacus Press, Cambridge, Mass.

28. Krijnen HG (1979) The flexible firm. Long Range Plann. 12, April, 63-75.

29. Lawrence $P$ and Lorsch $J$ (1967) Organization and Environment. Harvard School of Business Administration Press, Boston.

30. Maruyama M (1976) Towards cultural symbiosis. In Evolution and Consciousness: Human Systems in Transition (Edited by Jantsch $\mathrm{E}$ and Waddington $\mathrm{CH}$ ), pp. 198-213. Addison-Wesley, Reading, Mass.

31. Mascarenhas B (1982) Coping with uncertainty in international business. J. Int. Busin. Stud. 13, 87-98.

32. Maturana H and Varela F (1980) Autopoesis and Cognition: The Realization of the Living. Reidl, London.

33. Mintzberg H (1979) The Structuring of Organizations. Prentice-Hall, Englewood Cliffs, N.J.

34. Mintzberg $\mathrm{H}$ and Waters JA (1985) Of strategies, deliberate and emergent. Strategic Mgmt J. 6, 257-272.

35. Morgan G (1981) The schismatic metaphor and its implications for organizational analysis. Org. Stud. 2, 23-44.

36. Morgan G (1986) Images of Organization. Sage, Beverely Hills.

37. Pennings JM (1981) Strategically interdependent organizations. In Handbook of Organizational Design (Edited by Nystrom PC and Starbuck WH), Vol. 1, pp. 433455. Oxford University Press, New York.

38. Pfeffer J and Salancik GR (1978) The External Control of Organizations: A Resource Dependence Perspective. Harper \& Row, New York.

39. Prigogine I (1976) Order through fluctuation: self-organization and social system. In Evolution and Consciousness: Human Systems in Transition (Edited by Jantsch $\mathrm{E}$ and Waddington $\mathrm{CH}$ ), pp. 93-133. AddisonWesley, Reading, Mass.

40. Quinn JB (1980) Strategies for Change-Logical Incrementalism. Irwin, Homewood, Ill.

41. Quinn JB (1985) Managing innovation: controlled chaos. Harvard Busin. Rev. 63, 78-84.

42. Reichwald R and Behrbohm P (1983) Flexibilität als Eigenschaft productionswirtschaftlicher Systeme. $Z$. Betriebsk. 53, 831-853.

43. Scott BW (1965) Long-Range Planning in American Industry. American Management Association, New York.

44. Simon HA (1969) The Sciences of the Artificial. MIT Press, London.

45. Smith KK (1982) Philosophical problems in thinking about organizational change. In Change in Organizations: New Perspectives on Theory, Research, and Practice (Edited by Goodman PS et al.), pp. 316-374. Jossey-Bass, San Francisco.

46. Smith KK (1984) Rabbits, lynxes, and organizational transitions. In New Futures: The Challenge of Managing Corporate Transitions (Edited by Kimberly and Quinn JB), pp. 267-294. Dow Jones-Irwin, Homewood, Ill.

47. Snow RM (1993) The integration of systemic and interpretative thought. In International Systems Science Handbook (Edited by Delgado RR and Banathy BH), pp. 136-158. Systemic Publications, Madrid.

48. Steers RM (1975) Problems in the measurement of 
organizational effectiveness. Admin. Sci. $Q . \mathbf{2 0}$, 546-558.

49. Ulrich D and Lake D (1990) Organizational Capability. Wiley, New York.

50. Van de Bunt PAE (1987) Flexibel doch vasthoudend. Samsom, Alphen a/d Rijn.

51. Van Ham JC, Pauwe J and Williams ART (1987) Flexibiliteit en stabiliteit vanuit individu en organisatie (Flexibility and stability of individual and organization). In Arbeidsmarkt, Arbeidsorganisatie, Arbeidsverhoudingen, Sociaal Beleid (Edited by Buitendam A), pp. 74-90. Kluwer/NVP, Deventer.

52. Volberda HW (1992) Organizational Flexibility: Change and Preservation-A Flexibility Audit and Redesign Method. Wolters-Noordhoff, Groningen.

53. Volberda HW (1993) Flexible configuration strategies: managing change and preservation. 13th Annual International Conference 'Integrating Strategy' of the Strategic Management Society, Chicago.

54. Volberda HW and Cheah $\mathrm{H}$ (1993) A new perspective on entrepreneurship: a dialectic process of transformation within the entrepreneurial mode, type of flexibility and organizational form. In Research in Entrepreneur- ship (Edited by Klandt H), pp. 261-286. Avebury, Aldershot.

55. Weick KE (1979) The Social Psychology of Organizations. Addison-Wesley, Reading, Mass.

56. Weick KE (1982) Management of organizational change among loosely coupled elements. In Change in Organizations: New Perspectives in Theory, Research, and Practice (Edited by Goodman PS et al.), pp. 375-408. Jossey-Bass, San Francisco.

57. Weick KE (1993) Organizational redesign as improvisation. In Organizational Change and Redesign (Edited by Huber GP and Glick WH). Oxford University Press, New York.

58. Williamson OE (1975) Structural adaptations to environments. Acad. Mgmt Rev. 11, 921.

59. Zelenovic DM (1982) Flexibility-a condition for effective production systems. Int. J. Prod. Res. 20, 319-337.

ADDRESS FOR CORRESPONDENCE: $\mathrm{Dr} H W$ Volberda, Depart ment of Strategic Management and Business Environment, Rotterdam School of Management, Erasmus University, PO Box 1738, 3000 DR Rotterdam, The Netherlands. 